\title{
Differential and Statistical Approach to Partial Model Matching
}

\author{
Kehua Guo, Yongling Liu, and Guihua Duan \\ School of Information Science and Engineering, Central South University, Changsha 410083, China \\ Correspondence should be addressed to Kehua Guo; guokehua@csu.edu.cn
}

Received 2 December 2012; Accepted 23 December 2012

Academic Editor: Sheng-Yong Chen

Copyright (C) 2013 Kehua Guo et al. This is an open access article distributed under the Creative Commons Attribution License, which permits unrestricted use, distribution, and reproduction in any medium, provided the original work is properly cited.

\begin{abstract}
Partial model matching approaches are important to target recognition. In this paper, aiming at a 3D model, a novel solution utilizing Gaussian curvature and mean curvature to represent the inherent structure of a spatial shape is proposed. Firstly, a Point-Pair Set is constructed by means of filtrating points with a similar inherent characteristic in the partial surface. Secondly, a Triangle-Pair Set is demonstrated after locating the spatial model by asymmetry triangle skeleton. Finally, after searching similar triangles in a PointPair Set, optimal transformation is obtained by computing the scoring function in a Triangle-Pair Set, and optimal matching is determined. Experiments show that this algorithm is suitable for partial model matching. Encouraging matching efficiency, speed, and running time complexity to irregular models are indicated in the study.
\end{abstract}

\section{Introduction}

Partial model matching has important applications in many cases such as the vision registration for automatic 3D models [1-3], content-based image retrieval from image database [4], assembly and restoration for fractured object [5], docking of proteins in molecular biology [6], and other applications. During the past decade, the problem of finding a partial match between models attracted considerable attention. In many cases, models may be only partially visible because the occlusion or the sensor usually cannot scan all the data, so the goal of partial model matching is to find a transformation of the sample model to overlap a large portion of the object model.

This paper focuses on the research of a $3 \mathrm{D}$ partial model matching. In $3 \mathrm{D}$ space, the models can be represented as 3D surfaces. Therefore, finding a Euclidean geometry rigid transformation of the sample surface to overlap a large portion of the object surface is the goal of $3 \mathrm{D}$ partial model matching. In recent years, there has been lots of research on $3 \mathrm{D}$ partial surface matching. The literature $[7,8]$ proposed a scoring schema for $3 \mathrm{D}$ partial surface matching, but this paper did not demonstrate a universal and high-efficiency algorithm for the constructing of a scoring factor. The literature [9] proposed an approach based on the Hausdorff and Frehet distance for geometry structure matching. This approach can be well applied in the matching of points and line segments set, but it is unstable and cannot perform well in the presence of noise, occlusion, and clutter. The literature [10] proposed a scoring schema for matching algorithm to a 3D incomplete object. The literature [11-13] proposed some matching algorithm using differential geometry to $3 \mathrm{D}$ partial surface matching. In addition, statistical descriptors [14], probabilistic framework [15], and curve analysis [16] were also applied to the recognition of $3 \mathrm{D}$ partial surface; these approaches indicated encouraging matching results for $3 \mathrm{D}$ points, line segments set, and mesh model; however, they would suffer from high computing complexity. It is worth mentioning that partial model matching has been successfully applied in video processing $[17,18]$.

Our work is motivated by the technique of scoring schema and statistical approaches. This technique, which uses the so-called scoring-function method, was originally introduced for 3D partial surface and volumetric matching. This idea has been applied in some pattern recognition problem [19] and achieved good results. The work proposed in this paper has been partly published in an international conference [20]. Here is a brief sketch of our algorithm.

(1) Represent the inherent characteristic of points in sample and object surface utilizing differential geometry method. 
(2) Classify point pair with a similar inherent characteristic form sample and object surface.

(3) Classify triangle pair with a similar shape feature and inherent characteristic based on point pairs and compute the geometry rigid transformation for every triangle pair.

(4) Use scoring function to measure the goodness of every transformation through applying it to other triangle pairs.

(5) Determine the optimal transformation with score of every transformation.

Experimental results show that our technique is most successful for the matching of irregular object surface. Encouraging matching efficiency, speed, and running time complexity to irregular models are indicated.

\section{Compute and Store the Curvature}

2.1. Represent the Spatial Surface Using Gaussian and Mean Curvature. According to differential geometry principle, curvature is the inherent characteristic of a spatial surface. Therefore, Gaussian curvature and mean curvature are employed for the representation of spatial surface in this paper.

In 3D Euclidean space, given a parametric surface defined as

$$
S(x, y)=[x y f(x, y)]^{T},(x, y) \in D
$$

where $X-Y$ is the reference plane in $3 \mathrm{D}$ space, $D$ is projection region of the surface to $X-Y$ plane, and $f(x, y)$ represents the distance from the surface to point $(x, y)$ in $X-Y$ plane.

Gaussian curvature $K$ and mean curvature $H$ can be computed according to the following formulas [18]:

$$
\begin{gathered}
K=\frac{f_{x x} f_{y y}-f_{x y}^{2}}{\left(1+f_{x}^{2}+f_{y}^{2}\right)^{2}}, \\
H=\frac{\left(1+f_{x}^{2}\right) f_{y y}+\left(1+f_{y}^{2}\right) f_{x x}-2 f_{x} f_{y} f_{x y}}{2\left(1+f_{x}^{2}+f_{y}^{2}\right)^{3 / 2}} .
\end{gathered}
$$

For a digital range image surface, approximations can be computed by a local polynomial fitting approach, and $n \times n$ operator is usually utilized to the convolution operation with the original range image:

$$
\begin{gathered}
f_{x}=D_{x} * f, \quad f_{y}=D_{y} * f \\
f_{x x}=D_{x x} * f, \quad f_{x y}=D_{x y} * f, \quad f_{y y}=D_{y y} * f,
\end{gathered}
$$

where $D$ is $n \times n$ operator. For $n=7$, the parameters can be computed as follows:

$$
\begin{aligned}
& D_{x}=d_{0} d_{1}^{T}, \quad D_{y}=d_{1} d_{0}^{T}, \\
& D_{x x}=d_{0} d_{2}^{T}, \quad D_{y y}=d_{2} d_{0}^{T}, \quad D_{x y}=d_{1} d_{1}^{T} \\
& d_{0}=\frac{1}{7}\left[\begin{array}{lllllll}
1 & 1 & 1 & 1 & 1 & 1 & 1
\end{array}\right]^{T} \\
& d_{1}=\frac{1}{28}\left[\begin{array}{lllllll}
-3 & -2 & -1 & 0 & 1 & 2 & 3
\end{array}\right]^{T} \\
& d_{2}=\frac{1}{84}\left[\begin{array}{lllllll}
5 & 0 & -3 & -4 & -3 & 0 & 5
\end{array}\right]^{T},
\end{aligned}
$$

where $d_{0}, d_{1}$, and $d_{2}$ are column vectors for window operator computing.

2.2. Generate the Point-Pair Set. After presenting the acquisition of Gaussian curvature $K$ and mean curvature $H$, in our algorithm, point pairs with similar curvature from sample and object surface will be classified, and then the point pairs will be stored into a Point-Pair Set. In order to measure the similarity between two points from sample and object surface, the definition of curvature distance is introduced as follows:

Definition 1. $\forall p_{i}^{1} \in S_{1}, p_{j}^{2} \in S_{2}$, curvature distance DC between point $p_{i}^{1}, p_{j}^{2}$ is

$$
\begin{aligned}
\operatorname{DC} & \left(p_{i}^{1}, p_{j}^{2}\right) \\
& =\frac{1}{2} \times\left(\frac{\left|K\left(p_{i}^{1}\right)-K\left(p_{j}^{2}\right)\right|}{\left|K\left(p_{i}^{1}\right)\right|+\left|K\left(p_{j}^{2}\right)\right|}+\frac{\left|H\left(p_{i}^{1}\right)-H\left(p_{j}^{2}\right)\right|}{\left|H\left(p_{i}^{1}\right)\right|+\left|H\left(p_{j}^{2}\right)\right|}\right) .
\end{aligned}
$$

However, in many cases, a lot of plane points, whose Gaussian curvature and mean curvature are zero, can exist in the surface. They cannot contribute to the matching but will occupy large computing and reduce the matching efficiency, even lead to an error matching. So that these plane points will be discarded before matching.

Definition 2. A Point-Pair Set named PS is a set defined as

$$
\begin{gathered}
\mathrm{PS}=\left\{\left(p_{i}^{1}, p_{j}^{2}\right) \mid p_{i}^{1} \in S_{1}, p_{j}^{2} \in S_{2},\right. \\
\mathcal{E C}=\frac{\left.\operatorname{mC}\left(p_{i}^{1}, p_{j}^{2}\right)\left\langle\varepsilon \mathcal{L},\left|K\left(p_{i}^{1}\right)\right|\right\rangle \sigma\right\}}{2}\left(\mathrm{DC}\left(p_{i}^{1}, p_{j}^{2}\right)\right)+\min \left(D C\left(p_{i}^{1}, p_{j}^{2}\right)\right) \\
\sigma=\frac{1}{n}\left(\sum_{i=1}^{n}\left|K\left(p_{i}^{1}\right)\right|\right) .
\end{gathered}
$$

Where $\varepsilon c$ is an average threshold to guarantee the similarity of $p_{i}^{1}$ and $p_{j}^{2}, \sigma$ is to discard plane points, and $N$ is the number of the points. 
The cardinality of PS could be large in special cases. For instance, cardinality of PS will be $\left|S_{1}\right| \times\left|S_{2}\right|$ when the sample and object surface are congruent sphere surface. A large Point-Pair Set will occupy a large amount of the storage space. Considering the quantity of curvature categories cannot exceed the quantity of pixel points in surface, a list can be employed to store the set.

\section{Match Algorithm}

3.1. Measure Similarity for Spatial Triangles. In order to perform the matching, the further work is to separately seek two asymmetrical triangles with similar shape features from the sample and object surface, and then we will design a set named "Triangle-Pair Set" to store these triangle pairs. A plane can be determined by three spatial points, so the asymmetrical triangle can be employed as the skeleton to uniquely confirm the location of a spatial surface. Therefore, we arbitrarily select three points from the object surface to form an asymmetrical triangle and then seek three corresponding points from sample surface to form another asymmetrical triangle, whose features of corresponding vertices and edges are similar to the previous triangle. The similarity of space triangles is measured by the definition as follows.

Definition 3. Consider $\forall\left(p_{1}^{1}, p_{1}^{2}\right),\left(p_{1}^{1}, p_{1}^{2}\right),\left(p_{1}^{1}, p_{1}^{2}\right) \in \mathrm{PS}$, $\left|p_{1}^{m} p_{2}^{m}\right|<\left|p_{1}^{m} p_{3}^{m}\right|<\left|p_{2}^{m} p_{3}^{m}\right|, m \in\{1,2\}$. The similar distance between $\Delta p_{1}^{1} p_{2}^{1} p_{3}^{1}$ and $\Delta p_{1}^{2} p_{2}^{2} p_{3}^{2}$ is

$$
\mathrm{DT}=\frac{1}{3} \times \sum_{1 \leq i<j \leq 3} \frac{|| p_{i}^{1} p_{j}^{1}|-| p_{i}^{2} p_{j}^{2}||}{\left|p_{i}^{1} p_{j}^{1}\right|+\left|p_{i}^{2} p_{j}^{2}\right|} .
$$

The absolute distance between $\Delta p_{1}^{1} p_{2}^{1} p_{3}^{1}$ and $\Delta p_{1}^{2} p_{2}^{2} p_{3}^{2}$ is

$$
\mathrm{DA}=2 \times \frac{\sum_{i=1}^{3}\left|p_{i}^{1} p_{i}^{2}\right|}{\sum_{1 \leq i<j \leq 3}^{3}\left(\left|p_{i}^{1} p_{j}^{1}\right|+\left|p_{i}^{2} p_{j}^{2}\right|\right)} .
$$

3.2. Compute Geometry Rigid Transformation. Suppose that $\Delta p_{1}^{1} p_{2}^{1} p_{3}^{1}$ and $\Delta p_{1}^{2} p_{2}^{2} p_{3}^{2}$ are two similar asymmetrical triangles, $\left(p_{i}^{1}, p_{i}^{2}\right)$, and $i \in\{1,2,3\}$ are corresponding points, we represent the transformation $T$ between $\Delta p_{1}^{1} p_{2}^{1} p_{3}^{1}$ and $\Delta p_{1}^{2} p_{2}^{2} p_{3}^{2}$ into a translation transformation $N$ and a rotation transformation $A$ :

$$
T\left(\Delta p_{1} p_{2} p_{3}\right)=A\left[\begin{array}{lll}
p_{1} & p_{2} & p_{3}
\end{array}\right]+N
$$

where $A=\left(n_{1} n_{2} n_{3}\right)^{T}$ is a rotation matrix and $N$ is a translation matrix.

We firstly shift the triangle centers to the origin of coordinate, so the transformation $T$ satisfies

$$
\left\lfloor\begin{array}{lll}
p_{1}^{2} & p_{2}^{2} & p_{3}^{2}
\end{array}\right\rfloor-N_{2}=A\left(\left\lfloor\begin{array}{lll}
p_{1}^{1} & p_{2}^{1} & p_{3}^{1}
\end{array}\right\rfloor-N_{1}\right)
$$

where $N_{1}, N_{2}$ is the center location matrix of $\Delta p_{1}^{1} p_{2}^{1} p_{3}^{1}$ and $\Delta p_{1}^{2} p_{2}^{2} p_{3}^{2}$.
Therefore, rotation matrix can be deduced as in the following formula:

$$
A=\left(\left\lfloor\begin{array}{lll}
p_{1}^{2} & p_{2}^{2} & \left.p_{3}^{2}\right\rfloor-N_{2}
\end{array}\right)\left(\left\lfloor\begin{array}{lll}
p_{1}^{1} & p_{2}^{1} & p_{3}^{1}
\end{array}\right\rfloor-N_{1}\right)^{-1} .\right.
$$

3.3. Generate Triangle-Pair Set. Similar triangle pairs from two surfaces will be stored in Triangle-Pair Set defined as follows:

Definition 4. A Triangle-Pair Set named TS is a set

$$
\begin{gathered}
\mathrm{TS}=\left\{\left(\Delta p_{1}^{1} p_{2}^{1} p_{3}^{1}, \Delta p_{1}^{2} p_{2}^{2} p_{3}^{2}, T\right) \mid\left(p_{i}^{1} p_{i}^{2}\right) \in \mathrm{PS}, \mathrm{DT}<\varepsilon t\right\} \\
\varepsilon t=\frac{\max (\mathrm{DT})+\min (\mathrm{DT})}{2},
\end{gathered}
$$

where $\varepsilon t$ is an average threshold to guarantee the similarity of $\Delta p_{1}^{1} p_{2}^{1} p_{3}^{1}$ and $\Delta p_{1}^{2} p_{2}^{2} p_{3}^{2} . T$ is the transformation from $\Delta p_{1}^{2} p_{2}^{2} p_{3}^{2}$ to $\Delta p_{1}^{1} p_{2}^{1} p_{3}^{1}$. So we can design the logic structure of Triangle-Pair Set as Figure 1.

3.4. Determine Optimal Transformation. The validity of every transformation will be tested through applying it to every other triangle pair in TS. For a specified transformation $T_{0}$ in PS, we define the scoring function as follows:

$$
\text { Score }\left(T_{0}\right)=\sum S\left(\Delta p_{1}^{1} p_{2}^{1} p_{3}^{1}, p_{1}^{2} p_{2}^{2} p_{3}^{2}, T_{0}\right) .
$$

Consider $\left(\Delta p_{1}^{1} p_{2}^{1} p_{3}^{1}, \Delta p_{1}^{2} p_{2}^{2} p_{3}^{2}\right)$ is an arbitrary similar triangle pair in PS, and function $S$ is defined as follows:

$$
\begin{aligned}
S & \left(\Delta p_{1}^{1} p_{2}^{1} p_{3}^{1}, \Delta p_{1}^{2} p_{2}^{2} p_{3}^{2}, T_{0}\right) \\
& = \begin{cases}1 & \mathrm{DA}\left(T_{0}\left(\Delta p_{1}^{1} p_{2}^{1} p_{3}^{1}\right), \Delta p_{1}^{2} p_{2}^{2} p_{3}^{2}\right)<\varepsilon a \\
0 & \text { others }\end{cases} \\
\varepsilon \boldsymbol{\varepsilon} a & =\frac{\max (\mathrm{DA})+\min (\mathrm{DA})}{2},
\end{aligned}
$$

where $\varepsilon a$ is an average threshold to determine whether $\Delta p_{1}^{1} p_{2}^{1} p_{3}^{1}$ can be transformed to $\Delta p_{1}^{2} p_{2}^{2} p_{3}^{2}$ by $T_{0}$. An optimal transformation $T_{P}$ is the transformation with maximum score satisfying

$$
\text { Score }\left(T_{P}\right)=\max (\operatorname{Score}(T)),
$$

where $T$ is an arbitrary transformation in TS.

\section{Experimental Results and Complexity Analysis}

4.1. Detect Partially Occluded Object. The goal of the first experiment is to detect the partially occluded object from a range image using the algorithm proposed in this paper. The image data is established referred to [21].

In Figures 2(a) and 2(b), two original objects (Duck and Venusm) in 3D space are demonstrated, and the surface is formed by pixel points. 


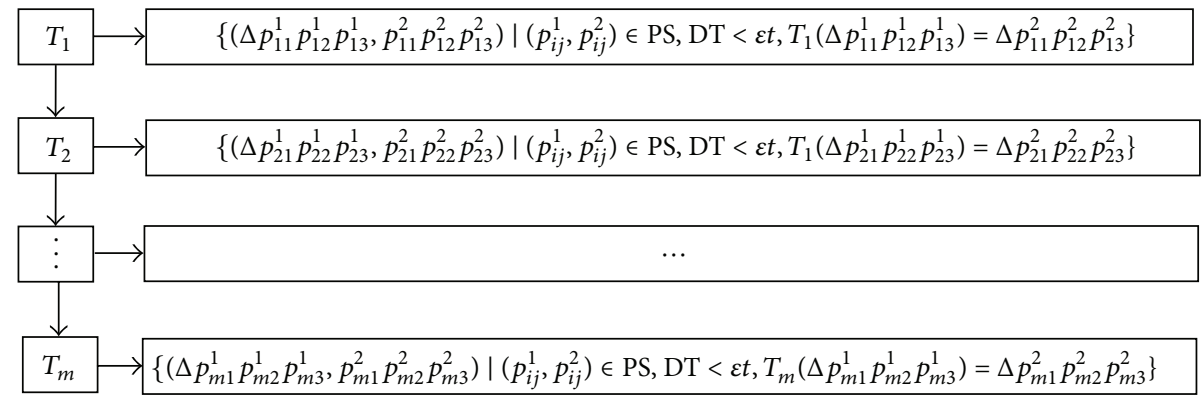

Figure 1: Logic structure of the Triangle-Pair Set.

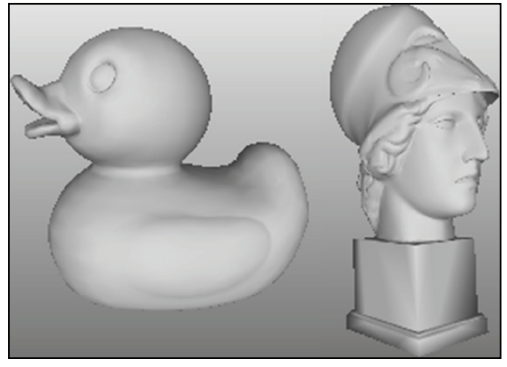

(a) Original object

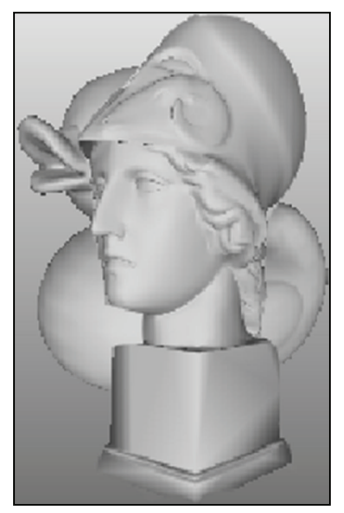

(c) Duck is partially occluded

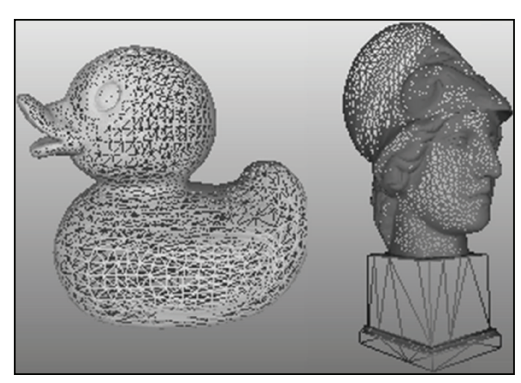

(b) 3D model of original object

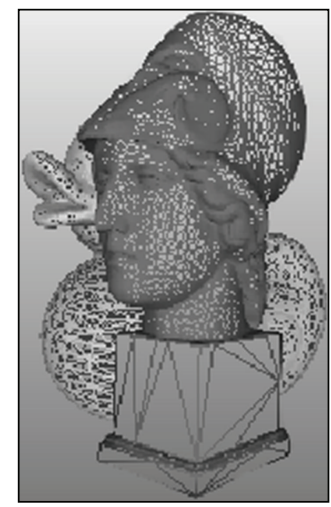

(d) 3D model of the scene

FIgURE 2: Two 3D original and partially occluded models.

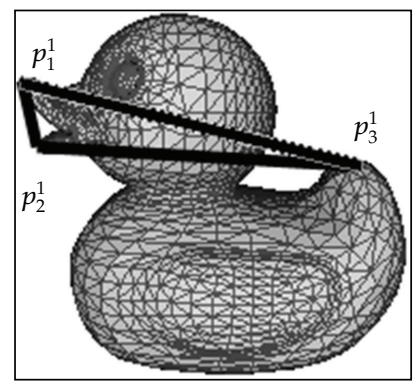

(a) Optimal triangle in original object

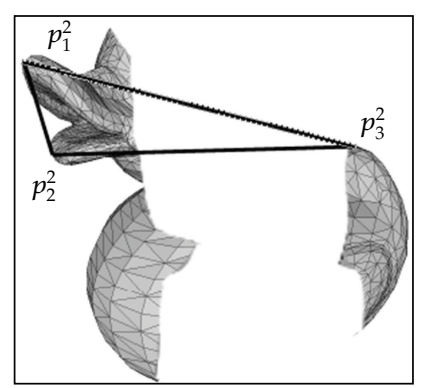

(b) Optimal triangle in occluded object

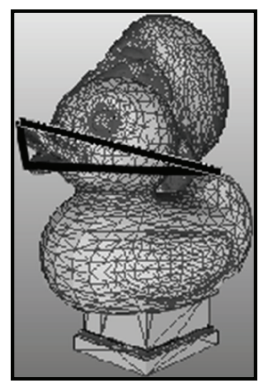

(c) Reconstruction result to occluded object

FIGURE 3: Detect partially occluded object. 


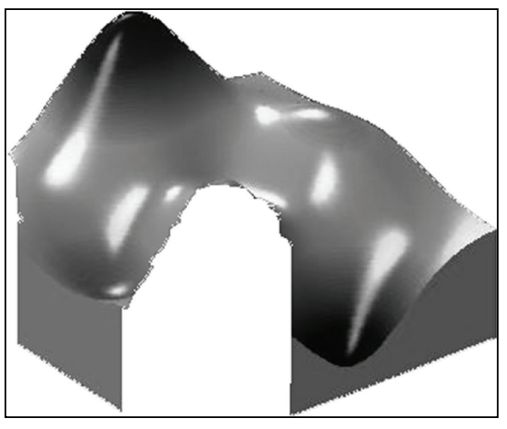

(a) Surface 1

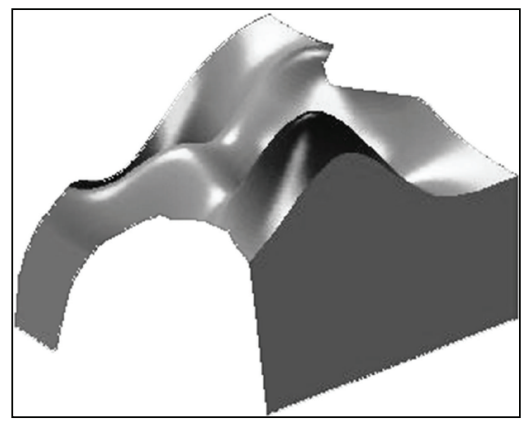

(b) Surface 2

FIgURE 4: Two partially similar surfaces.

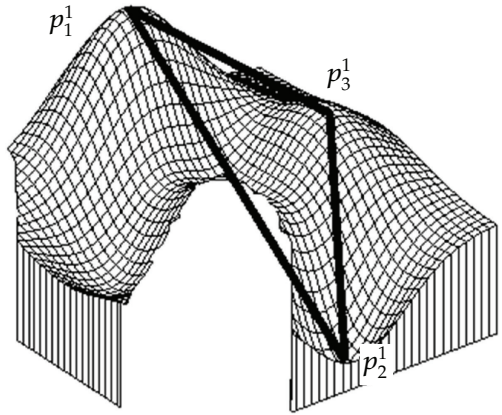

(a) Optimal triangle in surface 1



(b) Optimal triangle in surface 2

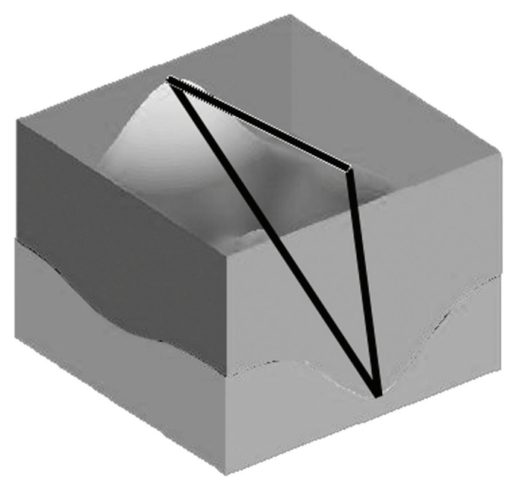

(c) Matching and reconstruction result

FIGURE 5: Partial surface matching of two similar surfaces.

TABLE 1: Parameters and computing results.

\begin{tabular}{|c|c|c|}
\hline \multirow{2}{*}{ Pixel point quantity } & \multicolumn{2}{|r|}{ Duck: 9253} \\
\hline & \multicolumn{2}{|r|}{ Venusm: 27595} \\
\hline Triangle pair for optimal transformation & $\begin{array}{r}p_{1}^{1}( \\
p_{2}^{1} \\
p_{3}^{1}(C\end{array}$ & $\begin{array}{lc}25,23,-15) & p_{1}^{2}(132,5,98) \\
72,4,-10) & p_{2}^{2}(83,-18,108) \\
8,-71,-23) & p_{3}^{2}(76,-89,82)\end{array}$ \\
\hline Transformation & $A=$ & {$\left[\begin{array}{ccc}1.2984 & -0.6040 & -0.6944 \\
-0.6401 & 1.2802 & -0.6401 \\
-0.6583 & -0.6763 & 1.3345\end{array}\right]$} \\
\hline
\end{tabular}




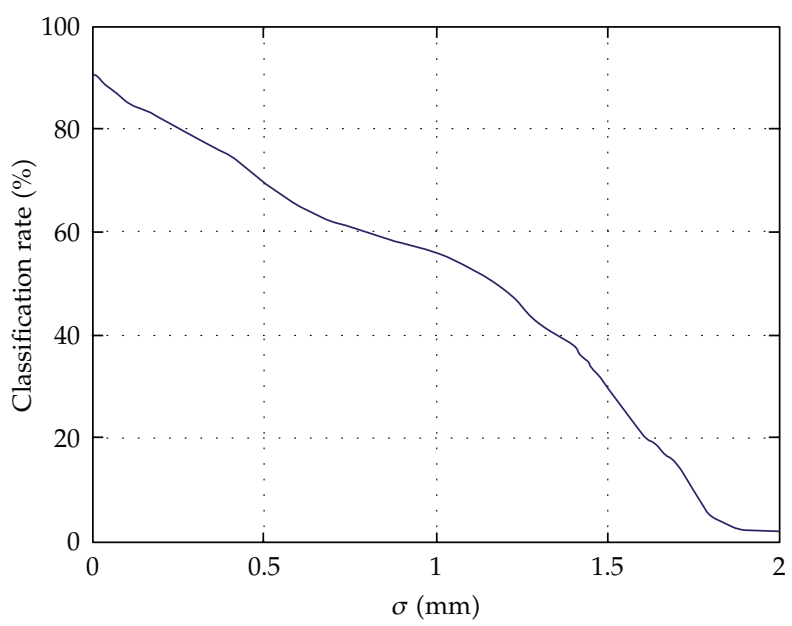

FIGURE 6: Classification rate when Gaussian noise increases.

TABle 2: Parameters and computing result.

\begin{tabular}{|c|c|c|c|c|}
\hline \multirow{2}{*}{ Pixel point quantity } & \multicolumn{4}{|c|}{ Object 1: 1215} \\
\hline & \multicolumn{4}{|c|}{ Object 2: 1452} \\
\hline \multirow{3}{*}{ Triangle pair for optimal transformation } & \multicolumn{4}{|c|}{$p_{1}^{1}(10,-5,87) \quad p_{1}^{2}(12,-69,18)$} \\
\hline & \multirow{2}{*}{\multicolumn{4}{|c|}{$\begin{array}{cc}p_{2}^{1}(8,-21,42) & p_{2}^{2}(25,-31,32) \\
p_{3}^{1}(29,-17,32) & p_{3}^{2}(92,-43,28)\end{array}$}} \\
\hline & & & & \\
\hline \multirow{3}{*}{ Transformation } & \multirow{3}{*}{$A=$} & 0.1028 & 1.7571 & -1.8599 \\
\hline & & -0.2024 & 1.1216 & -0.9191 \\
\hline & & 0.0996 & -2.8787 & 2.7791 \\
\hline
\end{tabular}

TABLE 3: Performance comparison of some algorithms.

\begin{tabular}{lcc}
\hline Algorithm & & Running time (ms) \\
& Sphere surface & Surface in Figure 3 \\
\hline Algorithm in this paper & 2635 & 758 \\
Algorithm in [9] & 1932 & 2575 \\
Algorithm in [10] & 2956 & 3548 \\
Algorithm in [11] & 1562 & 1652 \\
Algorithm in [14] & 1457 & 1238 \\
Algorithm in [16] & 3187 & 4572 \\
\hline
\end{tabular}

In Figure 2(c), the Duck is partially occluded by the Venusm. In order to find the location of the occluded object (Duck) in the 3D scene, we must compute an optimal transformation from the scene to the occluded object, then apply the transformation to the occluded object and reconstruct it in the 3D scene in Figure 2(d).

In this experimentation, the computing results are demonstrated in Table 1.

Optimal triangle in original object is demonstrated in Figure 3(a), and the optimal triangle in occluded object is illustrated in Figure 3(b). According to the result in Table 1, we apply the transformation to original object. The reconstruction result is shown in Figure 3(c).
4.2. Partial Surface Matching for Two Similar Objects. The second experimentation demonstrates our algorithm applied in a universal case. The two surfaces are partially similar, and the goal is to find a transformation to match the most similar part of the two surfaces.

The two surfaces with missing data in Figures 4(a) and 4(b) are generated by a Matlab function. In order to match the most similar part of the two surfaces, an optimal transformation from surface 1 to surface 2 needs to be computed.

The computing results in this experimentation are demonstrated in Table 2.

Optimal triangle in surface 1 is demonstrated in Figure 5(a), and the optimal triangle in surface 2 is illustrated 
in Figure 5(b). According to the results shown in Table 2, the transformation is applied to surface 1 and the matching and reconstruction result is demonstrated in Figure 5(c).

4.3. Complexity Analysis. Suppose that the two input sets have comparable sizes, we measure the computing complexity of the algorithm as a function of $N$ (the cardinality of the input sets); $k$ is the size of Point-Pair Set. Computing the curvature of every point takes $O(N)$ time. Preparing the Point-Pair Set can be executed with expected $O(N \log N)$ running time because we can sort the points based on curvature. In addition, seeking triangle pairs, computing and testing transformation based on Point-Pair Set will cost $O\left(k^{3}\right)$ running time. In total, the running time of the whole algorithm is $O\left(N+N \log N+k^{3}\right)$.

Obviously, the computing efficiency is mainly determined by the size of Point-Pair Set $k$. The quantity of point pairs will be smaller when the surface processes many different curvatures; in this case, our algorithm will cost less time at this time. So our algorithm is very suitable for the matching of irregular objects.

In order to explicitly describe the performance of our algorithm, we perform experimentations for objects with different shapes. Running times of some algorithms for $N=$ 900 are demonstrated in Table 3 (CPU: PIV2.0 GHZ, RAM: $1 \mathrm{~GB}$, Software: MATLAB 7.0).

We can see from Table 3 that our algorithm cannot indicate an encouraging matching efficiency for regular objects such as sphere surfaces.

4.4. Noise Robustness. The proposed work depends crucially on the Gaussian curvature and means curvature computation, which is notoriously sensitive to noise and local perturbation. In order to analyze the affection of noise to the match results, we perform an experimentation to verify the noise robustness of this algorithm. In this experimentation, we generate 100 similar surfaces by Matlab function, and Gaussian noise $(N(0, \sigma))$ is added to each image in the database and $\sigma$ changes from $0 \mathrm{~mm}$ to $2.0 \mathrm{~mm}$. We design the classification rate to represent the percentage of correct matching quantity in the database; the classification rates are shown in Figure 6 for various $\sigma$ values.

The result shows that the algorithm in this paper does not have good noisy robustness. At present, our approach can get encouraging matching efficiency and running time complexity in case of high signal to noise ratio. Therefore, the future work will concentrate on this problem.

\section{Conclusion}

In this paper, an approach for solving the $3 \mathrm{D}$ partial model matching is proposed. Firstly we utilize Gaussian curvature and mean curvature to represent the two object surfaces. Then curvature distance is defined to classify the point pair with similar curvature, and Point-Pair Set is employed to store point pairs. Secondly, we classify triangle pair with similar shape features from the two surfaces based on Point-Pair Set, similar distance is employed to measure the similarity of two triangles, and then the Triangle-Pair Set is generated. Finally, absolute distance and score function are proposed for measuring the goodness of a given transformation in Triangle-Pair Set, and a successful matching is determined based on the score of optimal transformation between the objects. Experimental results indicated our algorithm can be suitable for the matching of natural objects.

However, the efficiency of this approach would be reduced when the shape is regular and the robustness to noise is not very good. In future work, we will plan to explore several improvements of the algorithm, including the improvement of noise robustness, design of better distance definition, experimentation with other scoring functions, and further study of statistical approaches.

\section{Acknowledgments}

This work is supported by Research Fund for the Doctoral Program of Higher Education of China (20090162120069), Science and Technology Plan of Hunan (2009FJ3016), Postdoctoral Fund of Central South University, China Postdoctoral Science Foundation (2012M521552), and NSFC (61202341).

\section{References}

[1] L. Ritter, S. D. Reiz, D. Rothamel et al., "Registration accuracy of three-dimensional surface and cone beam computed tomography data for virtual implant planning," Clinical Oral Implants Research, vol. 23, no. 4, pp. 447-452, 2012.

[2] S. Y. Chen, H. Tong, and C. Cattani, "Markov models for image labeling," Mathematical Problems in Engineering, vol. 2012, Article ID 814356, 18 pages, 2012.

[3] S. Chen, Z. Wang, H. Tong et al., "Optimal feature matching for $3 \mathrm{D}$ reconstruction by combination of global and local information," Intelligent Automation and Soft Computing, vol. 17, no. 7, pp. 957-968, 2011.

[4] K. Bunte, M. Biehl, M. F. Jonkman, and N. Petkov, "Learning effective color features for content based image retrieval in dermatology," Pattern Recognition, vol. 44, no. 9, pp. 1892-1902, 2011.

[5] S. M. Tarte, H. Talib, M. A. G. Ballester, and F. Langlotz, "Evaluating partial surface matching for fracture reduction assessment," in Proceedings of the IEEE 3rd International Symposium on Biomedical Imaging: Nano to Macro, vol. 4, pp. 514-517, Arlington, Va, USA, April 2006.

[6] Y. Shibberu and A. Holder, "A spectral approach to protein structure alignment," ACM Transactions on Computational Biology and Bioinformatics, vol. 8, no. 4, pp. 867-875, 2011.

[7] G. Barequet and M. Sharir, "Partial surface and volume matching in three dimensions," IEEE Transactions on Pattern Analysis and Machine Intelligence, vol. 19, no. 9, pp. 929-948, 1997.

[8] G. Barequet and M. Sharir, "Partial surface matching by using directed footprints," Computational Geometry, vol. 12, no. 1-2, pp. 45-62, 1999.

[9] H. Alt, P. Bras, M. Godau, C. Knauer, and C. Wenk, "Computing the Hausdorff distance of geometric patterns and shapes," Discrete and Computational Geometry, Special IssueThe Goodman-Pollack-Festschrift, vol. 25, pp. 65-76, 2003. 
[10] N. J. Mitra, L. J. Guibas, and M. Pauly, "Partial and approximate symmetry detection for 3D geometry," ACM Transactions on Graphics, vol. 25, no. 3, pp. 560-568, 2006.

[11] R. Gal and D. Cohen-Or, "Salient geometric features for partial shape matching and similarity," ACM Transactions on Graphics, vol. 25, no. 1, pp. 130-150, 2006.

[12] S. Wang, Y. Wang, M. Jin, X. D. Gu, and D. Samaras, "Conformal geometry and its applications on 3D shape matching, recognition, and stitching," IEEE Transactions on Pattern Analysis and Machine Intelligence, vol. 29, no. 7, pp. 1209-1220, 2007.

[13] J. Tierny, J. P. Vandeborre, and M. Daoudi, "Partial 3D shape retrieval by Reeb pattern unfolding," Computer Graphics Forum, vol. 28, no. 1, pp. 41-55, 2009.

[14] U. Castellani, M. Cristani, S. Fantoni, and V. Murino, "Sparse points matching by combining 3D mesh saliency with statistical descriptors," Computer Graphics Forum, vol. 27, no. 2, pp. 643$652,2008$.

[15] A. Itskovich and A. Tal, "Surface partial matching and application to archaeology," Computers and Graphics, vol. 35, no. 2, pp. 334-341, 2011.

[16] H. Tabia, M. Daoudi, J. P. Vandeborre, and O. Colot, "A new 3D-matching method of nonrigid and partially similar models using curve analysis," IEEE Transactions on Pattern Analysis and Machine Intelligence, vol. 33, no. 4, pp. 852-858, 2011.

[17] S. Y. Chen, H. Tong, Z. Wang, S. Liu, M. Li, and B. Zhang, "Improved generalized belief propagation for vision processing," Mathematical Problems in Engineering, vol. 2011, Article ID 416963, 12 pages, 2011.

[18] S. Chen, Y. Wang, and C. Cattani, "Key issues in modeling of complex 3D structures from video sequences," Mathematical Problems in Engineering, vol. 2012, Article ID 856523, 17 pages, 2012.

[19] K. Guo, "A novel matching algorithm to 3D incomplete object," Journal of Computational Information Systems, vol. 7, no. 1, pp. 73-79, 2011.

[20] K. H. Guo and G. H. Duan, "3D partial surface matching using differential geometry and statistical approaches," Lecture Notes in Computer Science, vol. 7751, 2012.

[21] A. S. Mian, M. Bennamoun, and R. Owens, "Three-dimensional model-based object recognition and segmentation in cluttered scenes," IEEE Transactions on Pattern Analysis and Machine Intelligence, vol. 28, no. 10, pp. 1584-1601, 2006. 


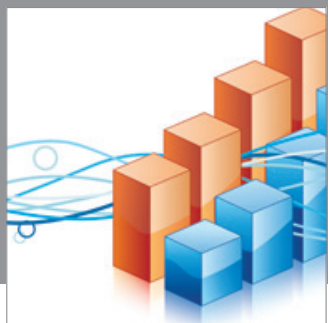

Advances in

Operations Research

mansans

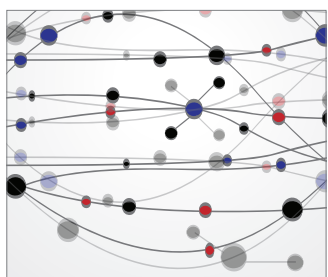

The Scientific World Journal
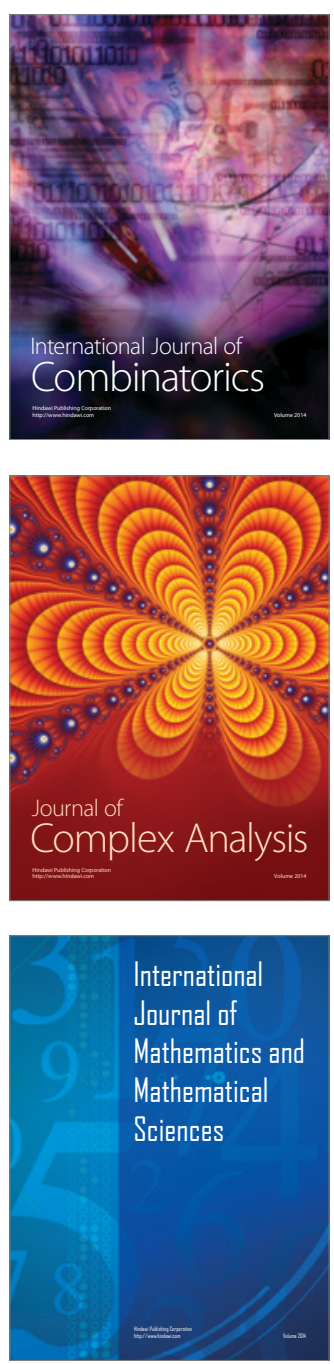
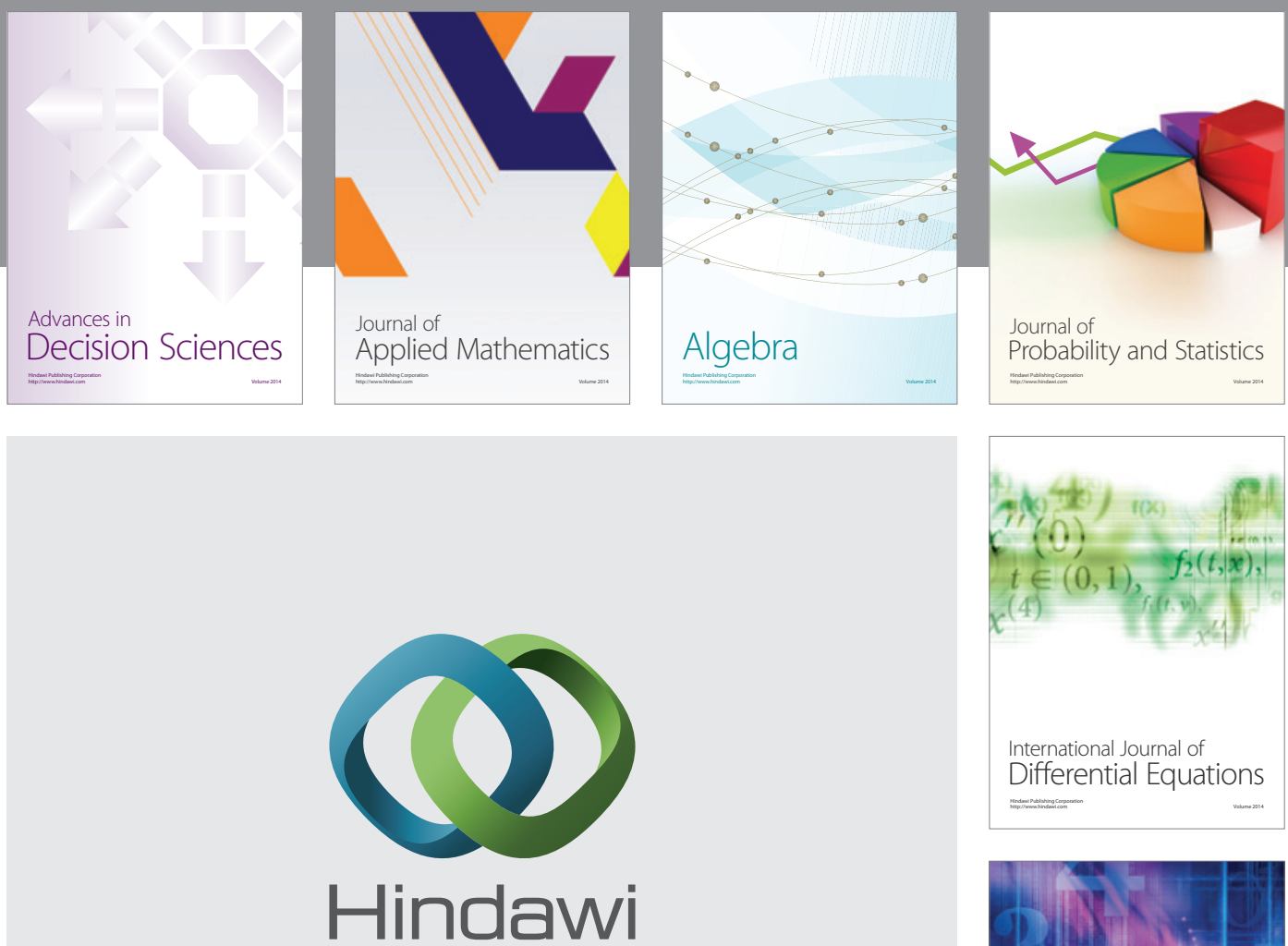

Submit your manuscripts at http://www.hindawi.com
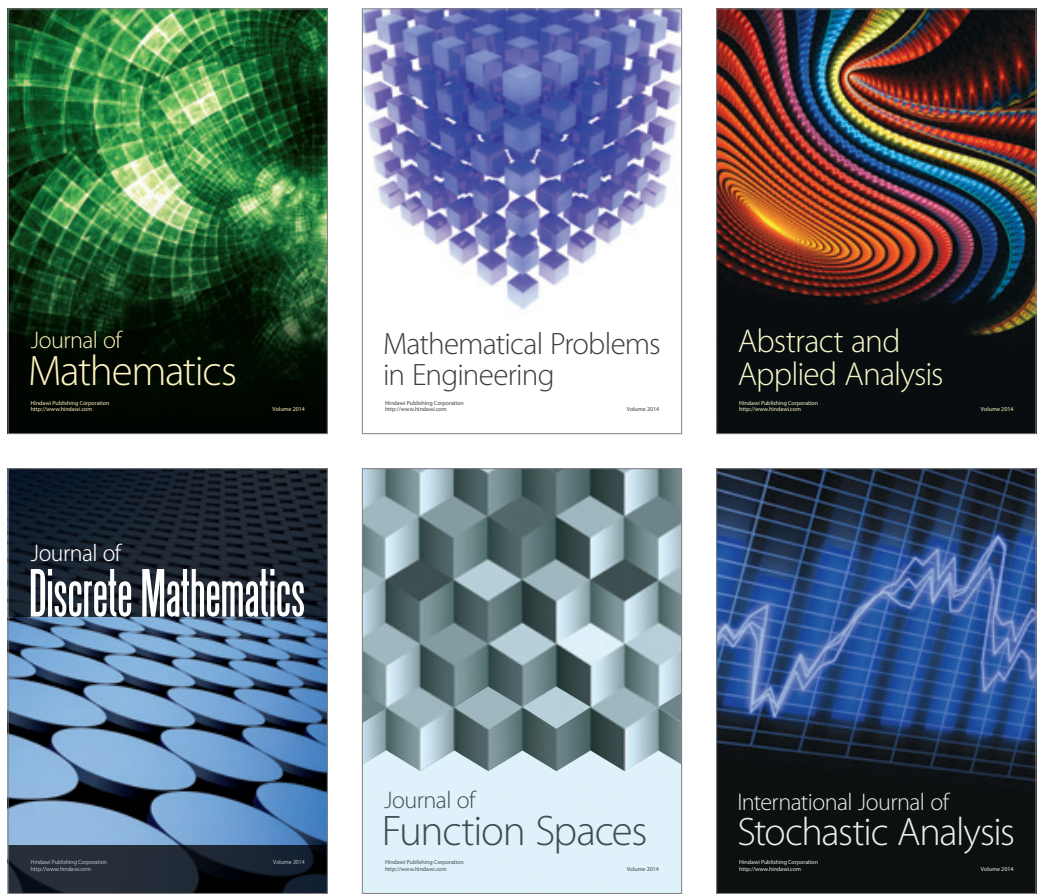

Journal of

Function Spaces

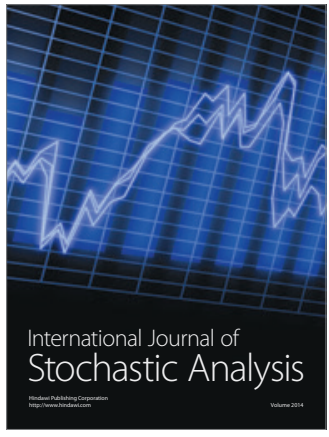

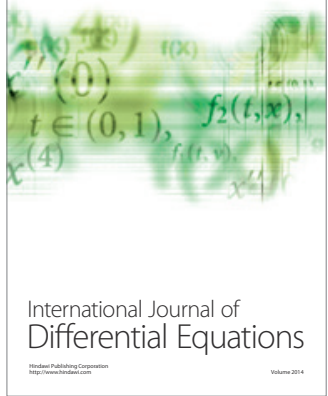
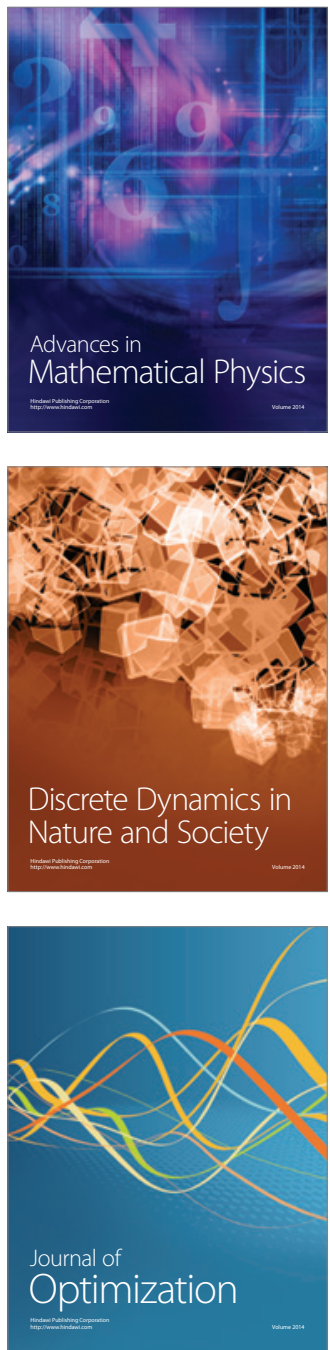